\title{
Simulation of wave propagation through aberrating layers of biological media
}

\author{
A.P. Berkhoff ${ }^{1}$, P.M. van den Berg ${ }^{2}$, J.M. Thijssen ${ }^{1}$ \\ ${ }^{1}$ University Hospital, 6500 HB Nijmegen, The Netherlands \\ ${ }^{2}$ Delft University of Technology, 2600 GA Delft, The Netherlands
}

\begin{abstract}
Two iterative methods for the calculation of acoustic reflection and transmission at a rough interface between two media are compared. The methods are based on a continuous version of the conjugate gradient technique. One method is based on plane-wave expansions while the other method is based on boundary integral equations and Green's functions. The methods are compared with regard to computational efficiency, rate of convergence, and residual error
\end{abstract}

\section{INTRODUCTION}

In echographic imaging, phase aberrations are often the major cause for image distortions if large array transducers are used. Phase aberrations are caused by irregular interfaces between tissue layers with a different propagation speed. In order to test the applicability and limits of phase aberration correction methods, ihe availability of an accurate computational tissue model is important. If the medium parameters within each layer are assumed to be constant, the computational problem can be reduced to the problem of finding the acoustic variables on the interfaces of the layered configuration. The discretization of the problem leads to a large number of unknowns. Due to the size of the numerical problem, iterative methods are essential. Iterative methods can lead to dramatically reduced storage requirements and total computation time. We will describe two iterative methods for the calculation of reflection and transmission at a rough interface between two media. Both methods are based on a continuous version of the conjugate gradient technique [1]. One method is based on plane-wave expansions [2] while the other method is based on boundary integral equations and free-space Green's functions [3]. Although the application deals with pulse-echo mode ultrasound, the domain of analysis is the frequency domain. An analysis in the frequency domain has the advantage that the strong frequency-dependent absorption and dispersive sound speed can be incorporated quite easily. Time-domain results can be obtained by analyzing the problem at several frequencies and subsequently calculating the inverse (temporal) Fourier transform. Wave propagation through random interfaces can be analyzed by evaluating a large number of interfaces [4].

\section{Formulation OF THE PROBLEM}

It is assumed that the roughness of the interface is a local deformation of an otherwise plane boundary at $z=0$, where a point in space is specified by its right-handed, orthogonal coordinates $x, y, z$. The analysis is carried out in the temporal frequency domain with angular frequency $\omega$ where the complex time factor $e^{-i \omega t}$ is suppressed. The two fluid-like media occupy the domains $\mathcal{D}_{1}$ and $\mathcal{D}_{2}$, respectively, and are assumed to be linear, homogeneous and isotropic with respective mass densities $\rho_{1}$ and $\rho_{2}$ and compressibilities $\kappa_{1}$ and $\kappa_{2}$. Furthermore, both media exhibit some losses and the real and imaginary parts of $\rho$ and $\kappa$ satisfy the KramersKronig causality relations. The interface is denoted by $\mathcal{S}$, and the pressure and the particle velocity vector by $P$ and $\mathrm{V}$, respectively. In $\mathcal{D}_{1}$, a source of finite extent generates a wave incident upon $\mathcal{S}$. The incident wave is denoted by $\left\{P_{i}, \mathrm{~V}_{i}\right\}$. The total field in $\mathcal{D}_{1}$ is written as the superposition of the incident field and the reflected field $\left\{P_{r}, V_{r}\right\}$. The total field in $\mathcal{D}_{2}$ is the transmitted field $\left\{P_{t}, V_{t}\right\}$.

\section{INTEGRAL EQUATION METHOD}

\section{Solution of the problem}

Employing the acoustic reciprocity theorem and assuming that the contribution from the parts of the contour integrals at infinity vanish, leads, at the interface $\mathcal{S}$, to the simultaneous integral equations [3]

$$
\begin{aligned}
& \frac{1}{2} P(\mathbf{x})-\int_{\mathbf{x}^{\prime} \in S}\left[\Gamma_{1}\left(\mathbf{x} \mid \mathbf{x}^{\prime}\right) \mathbf{V}\left(\mathbf{x}^{\prime}\right)+\Lambda_{1}\left(\mathbf{x} \mid \mathbf{x}^{\prime}\right) P\left(\mathbf{x}^{\prime}\right)\right] \cdot \nu\left(\mathbf{x}^{\prime}\right) d \mathbf{x}^{\prime}=P_{i}(\mathbf{x}) \\
& \frac{1}{2} P(\mathbf{x})+\int_{\mathbf{x}^{\prime} \in S}\left[\Gamma_{2}\left(\mathbf{x} \mid \mathbf{x}^{\prime}\right) \mathbf{V}\left(\mathbf{x}^{\prime}\right)+\Lambda_{2}\left(\mathbf{x} \mid \mathbf{x}^{\prime}\right) P\left(\mathbf{x}^{\prime}\right)\right] \cdot \nu\left(\mathbf{x}^{\prime}\right) d \mathbf{x}^{\prime}=0
\end{aligned}
$$

which relate the pressure $P$ at a point $\mathbf{x}$ on the interface $\mathcal{S}$ to contour integrals along $\mathcal{S}$ involving pressure and the normal component of the particle velocity. The outward normal of the surface $\mathcal{S}$ at $\mathbf{x}$, pointing into $\mathcal{D}_{1}$, is denoted by $\nu(\mathbf{x})$. The functions $\Gamma_{1}$ and $\Gamma_{2}$ contain the free-space Green's functions of $\mathcal{D}_{1}$ and $\mathcal{D}_{2}$, respectively. Similarly, $\Lambda_{1}$ and $\boldsymbol{\Lambda}_{2}$ contain the spatial derivatives of the free-space Green's functions of $\mathcal{D}_{1}$ and $\mathcal{D}_{2}$, respectively. Equations (1) and (2) constitute a system of two integral equations with two unknown quantities, viz., $P(\mathbf{x})$ and $\nu(\mathbf{x}) \cdot \mathbf{V}(\mathbf{x})$ on $\mathcal{S}$. It is noted that the integrals in the left-hand sides of (1) and (2) have to be interpreted as their principle values, i.e., the integrals are, when necessary, calculated by a limiting procedure that excludes the singularity at $\mathbf{x}=\mathbf{x}^{\prime}$ in a symmetrical manner. Once the solution to $P(\mathbf{x})$ and $\nu(\mathbf{x}) \cdot \mathbf{V}(\mathbf{x})$ has been found, the reflected field in $\mathcal{D}_{1}$ and the transmitted field in $\mathcal{D}_{2}$ follow from integrals over $\mathcal{S}$ [3]. The numerical solution to (1) and (2) can be obtained by discretizing the integral equations, evaluating the singular parts of the integrals, and solving the resulting system of linear equations by matrix inversion. This method will be called the Direct Integral Equation method (DIE). 


\section{Iterative solution of the problem}

The technique described in [1] is used to arrive at an iterative method for the solution of the two coupled integral equations. This method will be called the Iterative Integral Equation method (IIE). Normalizing the unknowns $P(x)$ and $V(\mathbf{x})=\mathbf{V}(\mathbf{x}) \cdot \nu(\mathbf{x})$ according to

$$
\begin{aligned}
P(\mathbf{x}) & =Z_{0}^{1 / 2} X_{P}(\mathbf{x}), \\
V(\mathbf{x}) & =Y_{0}^{1 / 2} X_{V}(\mathbf{x}),
\end{aligned}
$$

with $Z_{0}$ and $Y_{0}$ a reference impedance and admittance, respectively, the integral equations (1) and (2) can be written as

$$
\begin{aligned}
& Y_{1}(\mathbf{x})=\int_{\mathbf{x}^{\prime} \in S}\left[K_{1 P}\left(\mathbf{x}, \mathbf{x}^{\prime}\right) X_{P}\left(\mathbf{x}^{\prime}\right)+K_{1 V}\left(\mathbf{x}, \mathbf{x}^{\prime}\right) X_{V}\left(\mathbf{x}^{\prime}\right)\right] d \mathbf{x}^{\prime} \\
& Y_{2}(\mathbf{x})=\int_{\mathbf{x}^{\prime} \in S}\left[K_{2 P}\left(\mathbf{x}, \mathbf{x}^{\prime}\right) X_{P}\left(\mathbf{x}^{\prime}\right)+K_{2} v\left(\mathbf{x}, \mathbf{x}^{\prime}\right) X_{V}\left(\mathbf{x}^{\prime}\right)\right] d \mathbf{x}^{\prime}
\end{aligned}
$$

We assume the existence of an iterative procedure, in which $n$ steps have been carried out. The iterative procedure has led to the values $X_{P}^{(n)}(\mathbf{x})$ and $X_{V}^{(n)}(\mathbf{x})$. The integrated squared error after $n$ steps of iteration is

$$
E R R^{(n)}=\int_{\mathrm{x} \in S}\left(\left|F_{1}^{(n)}(\mathrm{x})\right|^{2}+\left|F_{2}^{(n)}(\mathrm{x})\right|^{2}\right) d \mathrm{x}
$$

in which

$$
\begin{aligned}
& F_{1}^{(n)}(\mathbf{x})=Y_{1}(\mathbf{x})- \\
& \int_{\mathbf{X}^{\prime} \in S}\left[K_{1 P}\left(\mathbf{x}, \mathbf{x}^{\prime}\right) X_{P}(n)\left(\mathbf{x}^{\prime}\right)+K_{1 V}\left(\mathbf{x}, \mathbf{x}^{\prime}\right) X_{V}^{(n)}\left(\mathbf{x}^{\prime}\right)\right] d \mathbf{x}^{\prime}, \\
& F_{2}^{(n)}(\mathbf{x})=Y_{2}(\mathbf{x})- \\
& \int_{\mathbf{X}^{\prime} \in S}\left[K_{2 P}\left(\mathbf{x}, \mathbf{x}^{\prime}\right) X_{P}^{(n)}\left(\mathbf{x}^{\prime}\right)+K_{2 V}\left(\mathbf{x}, \mathbf{x}^{\prime}\right) X_{V}^{(n)}\left(\mathbf{x}^{\prime}\right)\right] d \mathbf{x}^{\prime}
\end{aligned}
$$

with

In going from the $(n-1)$ st step to the $n$ th, we take

$$
X_{P, V}^{(n)}=X_{P, V}^{(n-1)}+\eta^{(n)} g_{P, V}^{(n)},
$$

where $\eta^{(n)}$ is a variational parameter to minimize $E R R^{(n)}$ and $g_{P, V}^{(n)}=g_{P, V}^{(n)}(\mathbf{x})$ are the conjugate gradient directions [5],

$$
g_{P, V}^{(n)}=s_{P, V}^{(n-1)}+\frac{A^{(n)}}{A^{(n-1)}} g_{P, V}^{(n-1)} \quad n \geq 2,
$$

while

$$
g_{P, V}^{(1)}=s_{P, V}^{(0)} .
$$

The directions $s_{P, V}^{(n)}=s_{P, V}^{(n)}(\mathbf{x})$ are the steepest-descent directions and $A^{(n)}$ is a scalar. It can be shown that the conjugategradient directions always give better convergence than the steepest-descent directions. Taking these directions, the error decreases at each iteration step according to

$$
E R R^{(n)}=E R R^{(n-1)}-\frac{\left|A^{(n)}\right|^{2}}{B^{(n)}}
$$

where $B^{(n)}$ is real and positive. We have a decrease of the error: $E R R^{(n)}<E R R^{(n-1)}$, provided $A^{(n)} \neq 0$. It can be shown [1] that $A^{(n)}$ is real and positive, unless $s_{P, V}^{(n-1)}$ vanishes. However, in the latter case, we have arrived at the exact solution in the iteration $n-1$.

$$
k_{z, 2}=\sqrt{\omega^{2} \rho_{2} \kappa_{2}-k_{x}^{2}-k_{y}^{2}} \quad \Re\left(k_{z, 2}\right), \Im\left(k_{z, 2}\right)>0 .
$$

The solution of the reflection/transmission problem can also be found by expanding the field in a sum (or integral) of plane waves [2]. We assume the existence of an iterative procedure, in which $n$ steps have been carried out. The iterative procedure has led to the plane-wave components $\phi_{r}^{(n)}$ and $\phi_{t}^{(n)}$ of the reflected and transmitted velocity potentials, respectively. The corresponding field values are

$$
\begin{aligned}
& \left\{P_{r}^{(n)}, \mathbf{V}_{r}^{(n)}\right\}=\iint_{-\infty}^{\infty}\left\{p_{r}^{(n)}, \mathbf{v}_{r}^{(n)}\right\} e^{i \mathbf{k}_{1}^{+} \cdot \mathbf{x}_{d k_{x}} d k_{y}, \quad}, \mathbf{x} \in D_{1} \\
& \left\{P_{t}^{(n)}, \mathbf{V}_{t}^{(n)}\right\}=\iint_{-\infty}^{\infty}\left\{p_{t}^{(n)}, \mathbf{v}_{t}^{(n)}\right\} e^{i \mathbf{k}_{2}^{-} \cdot \mathbf{x}_{d k_{x}} d k_{y},} \quad \mathbf{x} \in D_{2}
\end{aligned}
$$

where

$$
\begin{aligned}
& \left\{p_{r}^{(n)}, \mathbf{v}_{\tau}^{(n)}\right\}=i\left\{\omega \rho_{1}, \mathbf{k}_{1}^{+}\right\} \phi_{r}^{(n)} \\
& \left\{p_{t}^{(n)}, \mathbf{v}_{t}^{(n)}\right\}=i\left\{\omega \rho_{2}, \mathbf{k}_{2}^{-}\right\} \phi_{t}^{(n)}
\end{aligned}
$$

The plane-wave vectors $k_{1}^{+}$and $\mathbf{k}_{2}^{-}$indicate plane waves travelling away from $\mathcal{S}$ :

$$
\mathbf{k}_{1}^{+}=\left(k_{x}, k_{y},+k_{z, 1}\right)
$$

with

$$
k_{z, 1}=\sqrt{\omega^{2} \rho_{1} \kappa_{1}-k_{x}^{2}-k_{y}^{2}} \quad \Re\left(k_{z, 1}\right), \Im\left(k_{z, 1}\right)>0,
$$

and

The integrated squared error $E R R^{(n)}$ in the boundary conditions after $n$ steps of iteration is

$$
E R R^{(n)}=\int_{\mathbf{x} \in \mathcal{S}}\left(\left|F_{P}^{(n)}\right|^{2}+\left|F_{V}^{(n)}\right|^{2}\right) d \mathrm{x},
$$

in which the deviations $F_{P}^{(n)}=F_{P}^{(n)}(\mathbf{x}), F_{V}^{(n)}=F_{V}^{(n)}(\mathbf{x})$ are given by

$$
\begin{aligned}
& F_{P}^{(n)}=Y_{0}^{1 / 2}\left(P_{i}+P_{r}^{(n)}-P_{t}^{(n)}\right) \\
& F_{V}^{(n)}=Z_{0}^{1 / 2} \nu \cdot\left(\mathbf{V}_{i}+V_{r}^{(n)}-\mathbf{V}_{t}^{(n)}\right)
\end{aligned}
$$

In going from the $(n-1)$ st step to the $n$ th, we take

$$
\phi_{r, t}^{(n)}=\phi_{r, t}^{(n-1)}+\eta^{(n)} g_{r, t}^{(n)}
$$

where $\eta^{(n)}$ is a variational parameter to minimize $E R R^{(n)}$ and $g_{r, t}^{(n)}=g_{r, t}^{(n)}\left(k_{x}, k_{y}\right)$ are the conjugate gradient directions

$$
g_{r, t}^{(n)}=s_{r, t}^{(n-1)}+\frac{A^{(n)}}{A^{(n-1)}} g_{r, t}^{(n-1)} \quad n \geq 2,
$$

while

$$
g_{r, t}^{(1)}=s_{r, t}^{(0)} .
$$

As with the iterative integral equation method, the directions $s_{r, t}^{(n)}=s_{r, t}^{(n)}\left(k_{x}, k_{y}\right)$ represent the steepest-descent directions 
and $A^{(n)}$ is a scalar. It can be shown that the conjugategradient directions always give better convergence than the steepest-descent directions. Again we have a decrease of the error: $E R R^{(n)}<E R R^{(n-1)}$, provided $A^{(n)} \neq 0$. It can be shown [2] that $A^{(n)}$ is real and positive, unless $s_{r, t}^{(n-1)}$ vanishes. However, in the latter case, we have arrived at the exact solution in the iteration $n-1$.

\section{NUMERICAL IMPLEMENTATION}

Some details of the $2 \mathrm{D}$ implementation are presented now. All the integrations were performed as simple summations of the discrete function values. The integrations over $\mathbf{x}$ were replaced by integrations over the path length $l$ along the surface. Experiments with the step size showed that the calculations gave consistent results for a spatial integration step size $\Delta x \leq 0.2 \lambda$, where $\lambda$ is the smallest wavelength in both media. The singular parts of the integrals occuring in the integral equation methods (direct and iterative) were evaluated analytically [6]. The Hankel functions of the integral equation methods were calculated by using the results of section 9.4 in [7]. Although the latter expressions are intended for realvalued arguments, complex arguments with relatively small imaginary parts can also be used. The calculations were run on a $40 \mathrm{MHz}$ Sun-Sparc IPC in Matlab 4.2.

\section{ResUlTS}

\section{Configuration}

The irregular interface consists of a Hamming-weighted sinusoidal deviation from a plane interface, with a peak-topeak height of $2.3 \mathrm{~mm}$, i.e. about 8 wavelengths. The period of the interface irregularity is $7.7 \mathrm{~mm}$, i.e. about 25.5 wavelengths. An array transducer is positioned at depth $z=10 \mathrm{~mm}$ and the mean of the interface is at $z=0$. The transducer consists of 128 radiating elements, each having a width of $0.15 \mathrm{~mm}$, positioned at a grid distance of $0.2 \mathrm{~mm}$. The electronic focus is at depth $z=-40 \mathrm{~mm}$ (that is, at a distance of $50 \mathrm{~mm}$ from the array), in a medium with the parameters $\rho_{1}$ and $\kappa_{1}$. The array elements radiate with equal magnitudes of normal surface velocity. The frequency is $5 \mathrm{MHz}$ and the sound speeds are: $c_{1}=1550 \mathrm{~m} / \mathrm{s}$ and $c_{2}=1473 \mathrm{~m} / \mathrm{s}$, i.e. a sound speed contrast of 5 percent. The densities are $\rho_{1}=\rho_{2}=10^{3} \mathrm{~kg} / \mathrm{m}^{3}$. The attenuation is $0.5 \mathrm{~dB} / \mathrm{cm} / \mathrm{MHz}$. Note that due to the attenuation the compressibilities are complex valued.

\section{Numerical parameters}

The number of points on the surface $\mathcal{S}$ was 512 with a spacing of $\Delta x=0.2 \lambda$, with $\lambda=2 \pi / \omega \sqrt{\rho_{2} \kappa_{2}}$, the wavelength in medium 2 . The values for the reference admittance $Y_{0}$ and the reference impedance $Z_{0}$ were taken to be the geometric means of the admittances and impedances of both media. The number of plane waves for the IPW-method, i.e., the number of discrete $k_{x}$ values, was 512 . The maximum value of $\left|k_{x}\right|$ was chosen to be the largest value for which all the plane waves in both media are non-evanescent, i.e, $\max \left\{\left|k_{x}\right|\right\}=$ $\min \left\{k_{1}, k_{2}\right\}$.

\section{Performance}

The sound propagation through the irregular interface was analyzed with both the iterative integral equation method (IIE) and the iterative plane-wave method (IPW). In addition, the results of the direct integral equation method (DIE) were used as a reference. For a finite number of iterations the IIE-method always converges to the results obtained with the DIE-method. The beam pressure plot for the plane interface is shown in Fig. 1. The beam pressure plots show the incident pressure field $P_{i}(\mathbf{x})$ and the transmitted pressure field $P_{t}(\mathbf{x})$. The interface is shown as a dark line in the figures. The beam pressure plots for the irregular interface obtained with IIE (Fig. 2) and IPW (Fig. 3)) agree closely. For the accuracy we desire, the total computation time of IIE is larger than the computation time of IPW (Fig. 4). There are two reasons for this. Firstly, for the first few iteration steps, the convergence of IIE is not as fast as the convergence of IPW. Secondly, the computation of the Hankel functions for IIE requires much more time than the computation of the exponentials in IPW. However, the error obtained with the IPW-method reaches a steady value after a number of iterations, wheras the error obtained with IIE can be driven to an arbitrarily small value. For the numerical implementation described in this paper, the convergence becomes worse if evanescent waves are included. Evanescent waves would be required for approximating sound fields with arbitrarily small error. It should be possible to include evanescent waves in combination with more accurate integrations. In our opinion, however, the resulting decrease of the error is not worth the additional computational effort. Our interest is in simulation of interfaces of moderate roughness separating media with low contrast. Then, the final error value which can be obtained with IPW is sufficiently small.

\section{Conclusion}

Two iterative methods for the $2 \mathrm{D}$ simulation of wave propagation through aberrating interfaces were compared. The iterative plane-wave method seems to be sufficiently accurate for different media with low contrast and moderate surface roughness. The iterative integral equation method is more accurate but requires longer computation times.

\section{REFERENCES}

[1] P.M. van den Berg, "Iterative Computational Techniques in Scattering Based Upon the Integrated Square Error Criterion," IEEE Trans. Antennas Prop. AP-32, 1063-1071, 1984.

[2] A.P. Berkhoff, P.M. van den Berg, J.M. Thijssen, "Iterative Calculation of Reflected and Transmitted Acoustic Waves at a Rough Interface," submitted to IEEE Trans. Ultrason. Ferroel. Freq. Control.

[3] J.T. Fokkema and P.M. van den Berg, Seismic Applications of Acoustic Reciprocity. Amsterdam: Elsevier, 
[4] J.A. Ogilvy, Theory of wave scattering from random rough surfaces, IOP Publishing Ltd, London, 1991.

[5] P.M. van den Berg, "Iterative schemes based on minimization of a uniform error criterion," Chapter 2 in: T.K. Sarkar (Ed.), Application of Conjugate Gradient Method to Electromagnetics and Signal Analysis, PIER 5. Amsterdam: Elsevier, pp. 27-65, 1991.

[6] E.I. Thorsos, "The validity of the Kirchhoff approximation for rough surface scattering using a Gaussian roughness spectrum," J. Acoust. Soc. Am. 83, 78-92, 1988.

[7] M. Abramowitz, I.A. Stegun, Handbook of mathematical functions, (Dover Publications, Inc., New York, 1972).

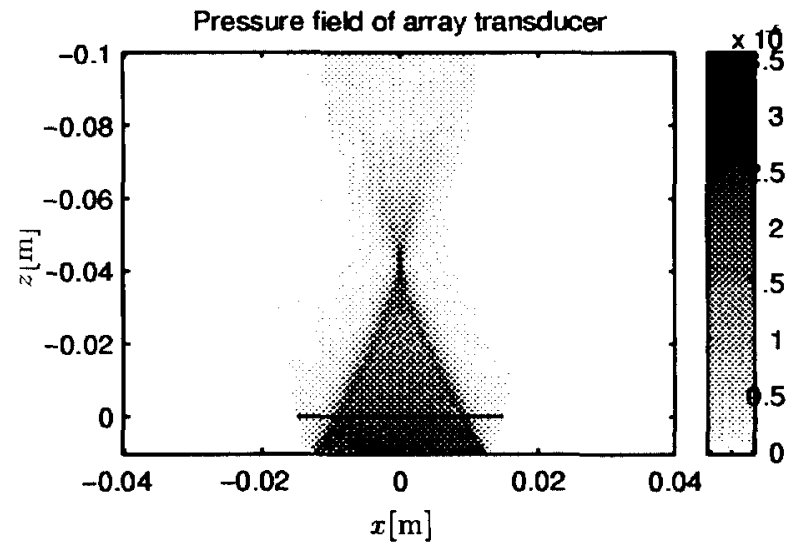

Fig. 1: Beam profile of array transducer for a plane interface separating two media, IPW-method. The array transducer is positioned at $z=0.01 \mathrm{~m}$ and radiates into a medium with parameters $c_{1}=1550 \mathrm{~m} / \mathrm{s}, \rho_{1}=10^{3} \mathrm{~kg} / \mathrm{m}^{3}$ at a frequency of of $5 \mathrm{MHz}$. The parameters of the second medium are: $c_{2}=$ $1473 \mathrm{~m} / \mathrm{s}, \rho_{2}=10^{3} \mathrm{~kg} / \mathrm{m}^{3}$. The attenuation in both media is $0.5 \mathrm{~dB} / \mathrm{cm} / \mathrm{MHz}$. The focus is at $z=-0.04 \mathrm{~m}$.

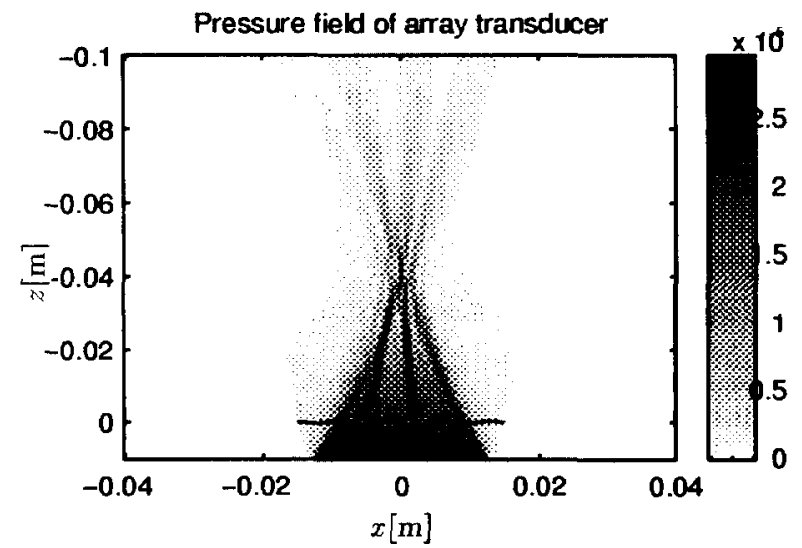

Fig. 2: Beam profile of array transducer for an irregular interface separating two media with parameters as in Fig. 1. IlE-method, initial guess: $X_{P, V}^{(0)}(\mathbf{x}) \equiv 0$, no. of iterations: $n=20$, resulting error: $\left(E R R^{(n)} / E R R^{(0)}\right)^{1 / 2}=0.013$.

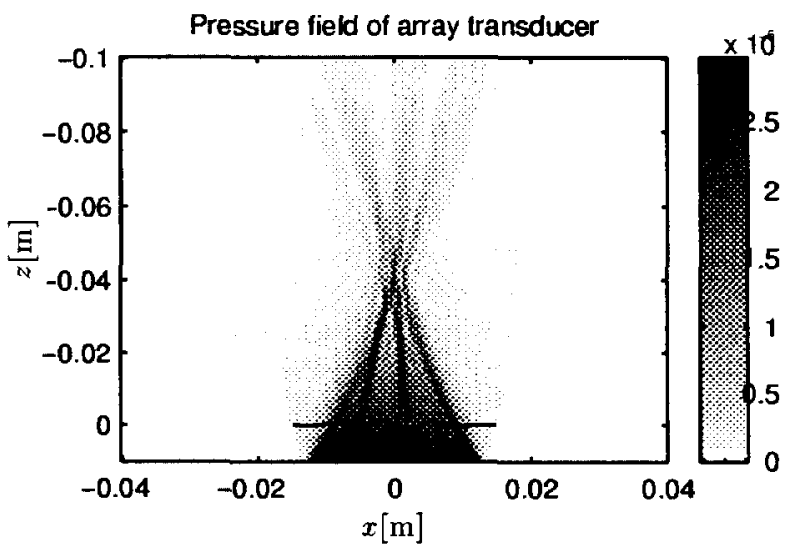

Fig. 3: Beam profile of array transducer for an irregular interface separating two media with parameters as in Fig. 1, IPW-method, initial guess: $\phi_{r, t}^{(0)}\left(k_{x}\right) \equiv 0$, no. of iterations: $n=10$, resulting error: $\left(E R R^{(n)} / E R R^{(0)}\right)^{1 / 2}=0.017$.

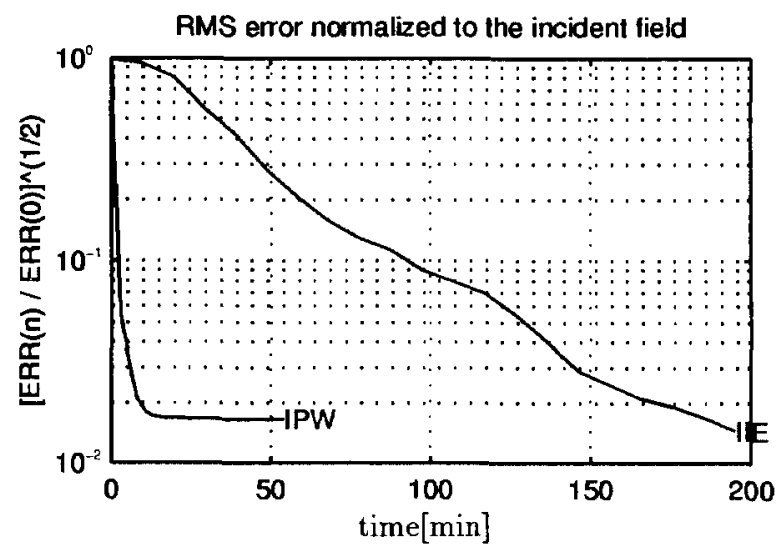

Fig. 4: Field error versus computation time on a $40 \mathrm{MHz}$ Sun-Sparc for the IIE-method and the IPW-method. 